\title{
Feasibility And Acceptability of Virtual Reality for Cancer Pain in People Receiving Palliative Care: A Randomised Cross- Over Study
}

\author{
Philip Austin ( $\sim$ paustin@hammond.com.au ) \\ Greenwich Hospital https://orcid.org/0000-0002-4627-5873 \\ Philip Siddall \\ HammondCare \\ Melanie Lovell \\ HammondCare
}

\section{Research Article}

Keywords: Virtual reality, cancer pain, palliative care, pain relief, presence

Posted Date: November 8th, 2021

DOI: https://doi.org/10.21203/rs.3.rs-847819/v1

License: @ (1) This work is licensed under a Creative Commons Attribution 4.0 International License. Read Full License

Version of Record: A version of this preprint was published at Supportive Care in Cancer on January 21st, 2022. See the published version at https://doi.org/10.1007/s00520-022-06824-x. 


\section{Abstract}

Context

Cancer pain is common and frequently undertreated; the development of innovative therapeutic options is needed.

Objectives

To determine the feasibility and preliminary effectiveness for larger randomised controlled trials of 3D head-mounted (HMD) virtual reality (VR) compared with 2D screens for managing cancer pain in adults.

Methods

Thirteen people receiving palliative and supportive care participated in a single-session randomised cross-over trial, after which they completed a qualitative semi-structured interview. We also compared the effects of 3D HMD VR and 2D screen applications on cancer pain intensity and levels of perceived presence. Feasibility was assessed with recruitment, completion rates and time required to recruit target sample.

Results

Although recruitment was slow, completion rate was high (93\%). Participants reported the intervention was acceptable and caused few side-effects. Although participants reported significantly reduced cancer pain intensity after 3D HMD VR (1.9 \pm $1.8, P=.003)$ and $2 \mathrm{D}$ screen applications $(1.5 \pm 1.6, \mathrm{P}=.007)$, no significant differences were found between interventions $(-.38 \pm 1.2,95 \% \mathrm{Cl}:-1.1-.29, \mathrm{P}=.23)$. Participants reported significantly higher levels of presence with the 3D HMD VR compared to $2 \mathrm{D}$ screen $(60.7 \pm$ SD 12.4 versus $34.3 \pm$ SD 17.1, mean 95\% Cl: 16.4-40.7, P=.001). Increased presence was associated with significantly lower pain intensity (mean $95 \% \mathrm{Cl}$ : $-.04--0.01, \mathrm{P}=0.02$ ).

Conclusions

We conclude that using HMD VR in palliative care pain settings is feasible and acceptable. In addition, virtual experiences provide relief of cancer pain. Further research is required to confirm the analgesic effects of VR in larger samples against appropriate control groups.

\section{Key Message}

This article describes the feasibility, acceptability and analgesic effects of virtual applications for people with cancer pain receiving palliative and supportive care. Results indicate that larger randomised clinical trials for the relief of cancer pain and other symptoms in palliative and supportive care settings are warranted.

\section{Introduction}

Over $50 \%$ of people with advanced cancer have pain and is common in out-of-hours care and hospitalisation [1]. Recent meta-analysis of pain in people with cancer shows pain prevalence at $66 \%$ in advanced metastatic disease compared to $39 \%$ after curative treatment and $55 \%$ during anticancer treatment [2]. While patients prefer care at home, pain is undertreated in about one third of patients in the community setting [3].

Cancer pain (CP) has peripheral, spinal and supraspinal neural mechanisms due to effects of disease and therapies. Psychological factors contribute where anxiety and depression are common and are shown to significantly increase pain severity and pain-related disability [4]. Pharmacological treatments for pain also have adverse effects on quality of life plus the potential interactions with anti-cancer drugs $[5,6]$. 
Despite guidelines [7] and best practice advocacy for pain management in palliative and supportive care settings [8], there remains a burden of unrelieved pain. A recent scoping review of pain management in advanced cancer settings, highlight recommended interventions are not always supported by evidence especially non-opioid pharmacology, invasive techniques and other non-pharmacological therapies. Given pain management in people with advanced cancer remains inadequate, innovative alternative therapeutic options such as virtual reality (VR) are needed.

VR is a computer-simulated 3D environment [9] and is shown to be an effective treatment for medical and psychological conditions $[10,11]$. While early VR systems used computer screens, current technologies include head-mounted devices (HMDs) with 3D-enabled glasses with additional sensory input devices including headphones for noise-cancelling, sound, head and/or body-tracking sensors and other input hardware such as joysticks[12]. These inputs form realistic multisensory experiences that surround users, described as 'real' or 'simulated' where the perceiver experiences a sense of presence, defined as an illusion of 'being there' [13]. Experimental investigations of VR analgesic mechanisms show that the degree of analgesic effect is dependent on the user's sense of presence in the virtual environment [14]. Encouragingly, meta-analysis shows no difference in effectiveness between specifically developed software and commercially available games using 3D environments [15].

VR presents an effective and harmless option for the management of pain in people with cancer. Currently, two studies using 3D-HMD VR applications show decreases in pain in cancer populations. To date, no randomised studies comparing 3D-HMD and 2D screen applications in people with CP exist. Evidence suggests that compared to 2D screen, 3D VR technologies are more realistic [16] where 3D perception of a VR scene is considered to give people a greater sense of presence.

The aim of our study is to evaluate feasibility in palliative care settings of a) recruitment for VR and data collection procedures in palliative care settings, b) acceptability of VR devices, c) completion rates of virtual interventions and d) provide information for future work in terms of estimating sample size. We further aimed to determine whether a) using a 3D-HMD VR device results in a significant reduction in CP compared to a 2D screen-based device running the same VR application and $b$ ) determine whether the level of presence in the virtual environment during the two interventions predicts the degree of analgesic effect in people with CP. We hypothesised 3D-HMD VR would result in significantly greater analgesia compared to 2D screen-based applications and that this is associated with greater levels of presence.

\section{Methods}

Study design

Using a within-subject, randomised cross-over feasibility trial, two sequential interventions were compared, one with 3D-HMD VR and one with a 2D screen using the same virtual environment in a convenience sample of 14 people with CP. Research staff (PA) generated random allocation for the first administered intervention using sequentially numbered, opaque sealed envelopes. Due to differences in appearance between the two interventions, participant and researcher blinding was not possible.

This study was an investigator-initiated feasibility trial funded by the Sydney Vital reference 201142, registered by the Australia New Zealand Clinical Trials Registry, number ACTRN-12619000568112p in April 2019. The Northern Sydney Local Health District Research Ethics Committee approved this single-site feasibility trial in March 2020, reference 2019/ETH12454. Study reporting complies with CONSORT guidelines [17].

- Feasibility was assessed by collecting data on the rate of completion of interventions (number of participants who completed both arms of the cross-over study). Feasibility was defined as $80 \%$ completion [18]. Feasibility of data collection methods was assessed by determining completion rates of trial outcome collection again defined as $80 \%$ completion.

- Acceptability of VR devices was determined using a semi-structured interview. 
Eligible participants were palliative and supportive care hospitalized and community-care patients. Inclusion criteria included adults, 18 years and older, a diagnosis of cancer, the presence of pain related to their cancer and/or cancer treatment over the previous 24 hours and at the time of study participation and a life expectancy of one month and over. Exclusion criteria included the presence of other types of pain more prominent at the time of study, psychiatric comorbidities not including anxiety, depression or stress, legally blind and a history of motion sickness. Participants were identified by hospital and community clinical staff, whereupon they were given participation information. Upon signed consent, participants completed a prevalidated case report form.

Study schedule

Baseline CP measures were taken for current, average, worst and least intensities over the previous 24 hours. Importantly, participants were instructed to rate only their CP. We compared differences in analgesic effect between 3D-VR HMD and 2D screen devices using the same environment over a duration of 15-minutes for each intervention. Primary CP scores were reported immediately after each intervention while additional NRS data was reported at five, 10- and 20-minutes post intervention. Lowest and average CP scores during interventions were also reported. To eliminate carryover effects from the first intervention, a washout period separated the two treatment periods (see Fig. 2). Here, participants were free to move around their surroundings. Because washout periods are required to be over five times the half-life of a treatment [19], we chose 60 minutes, based on data showing that pain is significantly reduced immediately after VR exposures but lasts no longer than 10 minutes after VR exposure [20]. Participants were required to report headset discomfort and cyber-sickness (includes symptoms of nausea, headache and vertigo) during or after using both applications.

\section{VR interventions}

\section{D HMD VR application}

The Oculus Rift@ headset is commercially available, relatively inexpensive and utilised for VR studies in medical research [21]. For this study, participants viewed a 3D virtual alpine meadow experience called Nature Trek®. Prior to use, participants were instructed on the use of a hand-held joystick, how to move around the environment and make full use of the $360^{\circ}$ scene. To avoid cybersickness, this audio-visual experience was non-interactive [22]. The VR headset was calibrated for participants' eyesight in addition to advice on motion sickness prevention during VR such as reducing speed and/or reducing head movement (Supplementary Fig. 1).

\section{D screen application}

The same application including audio inputs was run on a 17.3-inch Alienware ${ }^{\circledR}$ laptop screen with the participant seated in the same position. This allowed for reliable comparisons between the effects of 3D VR and 2D screen experiences.

\section{Outcome measures}

Pain numerical rating scale (NRS). Participants completed an 11-point pain NRS after each intervention where they reported levels of pain intensity after each intervention, and average and lowest pain intensities during each intervention. The 11-point pain NRS is a reliable and valid measure where meta-analysis shows extensive use across pain populations [23].

Edmonton Symptom Assessment System (ESAS): Participants completed the SAS at baseline and immediately after each VR session. The ESAS is a validated self-reporting tool used in cancer populations consisting of nine visual analogue scales measuring pain, activity, nausea, depression, anxiety, drowsiness, appetite, well-being and shortness of breath in palliative care settings [24]. 
Australian-modified Karnofsky Performance Status (AKPS): For overall performance status at baseline, we completed the AKPS at baseline only. This is a validated measure of a patient's overall performance status using a single score between 10 and 100 [25].

iGroup Presence Questionnaire (IPQ): To investigate levels of presence during each VR session, participants completed the IPQ after each application. The IPQ is a valid and reliable seven-point Likert scale for measuring spatial awareness, levels of involvement and experienced realism in virtual environments ranging from 0 (Not at all) to 6 (Very much) [26].

An adapted semi-structured interview guide designed by Pedroli and colleagues [27] was used to explore participant perceptions of 2D and 3D platforms. The interview focused on four primary topics: usability, presence, cybersickness and VR expectations. For the usability topic, questions were based upon sub-themes of user application, learning and satisfaction. Concerning the presence topic, questions were based upon sub-themes of spatial awareness, engagement and VR realism. Cybersickness and expectations questions related to symptoms felt during and after each application, expected amount of VR use in the future and finally, the use of VR for any other symptoms or complaints (Table 1).

Table 1

Topics, sub-topics and questions of semi-structured interview

(adapted from Pedroli et al 2018)

\begin{tabular}{|c|c|c|}
\hline Topic & Subtopic & Questions \\
\hline \multirow[t]{6}{*}{ Usability } & \multirow[t]{3}{*}{ Application } & 1. How did you find using the hand-held device? \\
\hline & & 2. How did you find moving around the virtual environment? \\
\hline & & $\begin{array}{l}\text { 3. Were there any technical problems with the headset and/or hand-held devices during } \\
\text { the VR session? }\end{array}$ \\
\hline & Learning & $\begin{array}{l}\text { 4. Did you ask or need help to understand how to use the VR headset and/or hand-held } \\
\text { devices during the VR session? }\end{array}$ \\
\hline & \multirow[t]{2}{*}{ Satisfaction } & 5. What did you like about the virtual environment? \\
\hline & & 6. How did you feel during the VR session? \\
\hline \multirow[t]{5}{*}{ Presence } & \multirow{2}{*}{$\begin{array}{l}\text { Spatial } \\
\text { awareness }\end{array}$} & 7. How immersed did you feel in the virtual environment? \\
\hline & & 8. How much control did you have when moving and observing the virtual environment? \\
\hline & \multirow[t]{2}{*}{ Engagement } & 9. What did you think about during the VR session? \\
\hline & & 10. Did you get distracted by noises outside the virtual environment? \\
\hline & Realism & 11. How did you find the virtual environment, realistic or too artificial? \\
\hline \multirow{2}{*}{$\begin{array}{l}\text { Cyber } \\
\text { sickness }\end{array}$} & \multirow{2}{*}{$\begin{array}{l}\text { Physical } \\
\text { effects }\end{array}$} & 12. Did you feel ill during the VR session? \\
\hline & & $\begin{array}{l}\text { 13. If yes, did you have nausea, dizziness or other physical symptoms during the VR } \\
\text { session? }\end{array}$ \\
\hline \multirow[t]{2}{*}{ Expectations } & Future use & 14. How often would you use virtual reality for pain relief in the future? \\
\hline & Other uses & $\begin{array}{l}\text { 15. Would you use virtual reality for any other complaints such as negative feelings } \\
\text { and/or physical symptoms? }\end{array}$ \\
\hline
\end{tabular}

\section{Data analysis}

Data analyses were performed using SPSS Statistics 24 [28]. Descriptive statistics were drawn from demographic and clinical features of the sample including age, years since cancer diagnosis, performance status, cancer stage, duration of $\mathrm{CP}$ and type of cancer. We used linear mixed model analysis for repeated measures with post 3D-HMD VR and 2D screen pain 
and presence scores as dependent variables for post 3D-HMD VR and 2D screen time points. These regressions included a factor for the condition (3D-HMD VR and 2D screen), the sequence (randomised sequence of conditions between subjects) and time (randomised sequence of conditions within subjects), where sequence was modelled as a random effect. The analysis controlled for baseline (pre-randomisation) pain intensity in models where post-intervention pain intensity was the dependent variable [29]. We also used paired sample T-test to compare pre-post mean difference scores for pain, presence and ESAS items within each treatment condition.

\section{Results}

Between July 82020 and May 13 2021, eight patients in community care and 15 in hospital care with cancer pain were contacted to participate in this study of whom 14 were enrolled into the study. The main reason for slow recruitment was the presence of the COVID-19 pandemic. One participant withdrew during the study due to excess levels of non-cancer-related low back pain, giving a 93\% study completion rate. Table 2 shows participants' demographic characteristics including age, gender, levels of cancer pain over the previous 24 hours, type of primary cancer, type(s) of cancer therapy, types of cancer care and CP location. Baseline, post intervention, mean differences and p-values for 3D HMD VR and 2D screen for CP intensity and ESAS items are shown in Table 3. Figure 3 shows recruitment and dropout of participants during the study.

Table 2 Baseline characteristics of 14 participants with cancer pain receiving palliative care. 


\begin{tabular}{|c|c|}
\hline Baseline Characteristics & Mean (SD) \\
\hline Age & $71.1(14.3)$ \\
\hline Years with cancer & $6.0(4.2)$ \\
\hline Months with cancer pain & $15.8(9.4)$ \\
\hline AKPS score & $66.9(14.9)$ \\
\hline \multicolumn{2}{|c|}{ Cancer pain levels over previous 24 hours $(0-10)$} \\
\hline - Average & $4.8(2.2)$ \\
\hline - Worst & $6.1(2.5)$ \\
\hline \multirow[t]{2}{*}{ - Least } & $2.7(2.1)$ \\
\hline & Number \\
\hline \multicolumn{2}{|l|}{ Gender } \\
\hline - Female & 6 \\
\hline - Male & 8 \\
\hline \multicolumn{2}{|l|}{ Patient-type } \\
\hline - Inpatient & 11 \\
\hline - Outpatient & 3 \\
\hline \multicolumn{2}{|l|}{ Primary cancer } \\
\hline - $\quad$ ENT & 2 \\
\hline - Neuroendocrine & 1 \\
\hline - Pancreatic & 2 \\
\hline - Colorectal & 2 \\
\hline - $\quad$ Breast & 1 \\
\hline - Prostate & 2 \\
\hline - Cervical & 1 \\
\hline - $\quad \mathrm{AML}$ & 1 \\
\hline - Liposarcoma & 1 \\
\hline - Ovarian & 1 \\
\hline \multicolumn{2}{|l|}{ Stage of illness } \\
\hline - $\quad$ Stage 4 & 14 \\
\hline \multicolumn{2}{|l|}{ Cancer therapy (past and current) } \\
\hline - Chemotherapy & 2 \\
\hline - Radiotherapy & 4 \\
\hline - Combined & 8 \\
\hline \multicolumn{2}{|l|}{ Type of care } \\
\hline - Symptom management & 11 \\
\hline - End of life care & 3 \\
\hline \multicolumn{2}{|l|}{ Cancer pain location } \\
\hline - Face/throat & 2 \\
\hline - Abdomen & 2 \\
\hline - $\quad$ Spine/ribs & 3 \\
\hline - $\quad$ Pelvis & 6 \\
\hline - $\quad$ Hips & 1 \\
\hline \multicolumn{2}{|l|}{ Cancer pain consistency } \\
\hline - Constant & 8 \\
\hline - Intermittent & 6 \\
\hline
\end{tabular}

Abbreviation - Australian-modified Karnofsky Performance Status (AKPS) 
Table 3

Baseline and post 3D HMD VR and 2D computer screen mean scores, mean differences, confidence intervals and $p$-values for palliative care symptoms using paired T-Test analysis

\begin{tabular}{|c|c|c|c|c|c|c|c|c|c|c|}
\hline \multirow[t]{2}{*}{ Mean (SD) } & \multirow[b]{2}{*}{ Baseline } & \multirow[b]{2}{*}{$\begin{array}{l}\text { During and } \\
\text { post } \\
\text { treatment }\end{array}$} & \multicolumn{4}{|c|}{ 3D Head mounted VR } & \multicolumn{4}{|c|}{ 2D computer screen } \\
\hline & & & $\begin{array}{l}\text { Mean } \\
\text { (SD) }\end{array}$ & $\begin{array}{l}\text { Mean } \\
\text { diff } \\
\text { (SD) }\end{array}$ & $\begin{array}{l}95 \% \mathrm{Cl} \text { of } \\
\text { difference }\end{array}$ & $\begin{array}{l}\mathrm{P}- \\
\text { value }\end{array}$ & $\begin{array}{l}\text { Mean } \\
\text { (SD) }\end{array}$ & $\begin{array}{l}\text { Mean } \\
\text { diff } \\
\text { (SD) }\end{array}$ & $\begin{array}{l}95 \% \mathrm{Cl} \text { of } \\
\text { difference }\end{array}$ & $\begin{array}{l}\mathrm{P} \text { - } \\
\text { value }\end{array}$ \\
\hline \multirow{6}{*}{$\begin{array}{l}\text { Cancer } \\
\text { pain } \\
\text { intensity }\end{array}$} & $3.6(1.4)$ & $\begin{array}{l}\text { Average } \\
\text { pain during }\end{array}$ & $\begin{array}{l}1.6 \\
(1.6)\end{array}$ & $\begin{array}{l}2.0 \\
(1.7)\end{array}$ & $.98-3.1$ & .001 & $\begin{array}{l}1.9 \\
(1.7)\end{array}$ & $\begin{array}{l}1.7 \\
(1.4)\end{array}$ & $.87-2.5$ & .001 \\
\hline & & $\begin{array}{l}\text { Least pain } \\
\text { during }\end{array}$ & $\begin{array}{l}.9 \\
(1.5)\end{array}$ & $\begin{array}{l}2.7 \\
(1.8)\end{array}$ & $1.6-3.8$ & .0002 & $\begin{array}{l}1.2 \\
(1.8)\end{array}$ & $\begin{array}{l}2.5 \\
(1.6)\end{array}$ & $1.5-3.4$ & .0001 \\
\hline & & $\begin{array}{l}\text { Immediately } \\
\text { post }\end{array}$ & $\begin{array}{l}1.8 \\
(1.5)\end{array}$ & $\begin{array}{l}1.9 \\
(1.8)\end{array}$ & $2.9-3.8$ & .003 & $\begin{array}{l}2.2 \\
(1.8)\end{array}$ & $\begin{array}{l}1.5 \\
(1.6)\end{array}$ & $2.4-3.3$ & .007 \\
\hline & & $\begin{array}{l}5 \text { minutes } \\
\text { post }\end{array}$ & $\begin{array}{l}2.3 \\
(1.5)\end{array}$ & $\begin{array}{l}1.3 \\
(1.8)\end{array}$ & $.19-2.4$ & .025 & $\begin{array}{l}1.9 \\
(1.9)\end{array}$ & $\begin{array}{l}1.8 \\
(1.9)\end{array}$ & $2.3-3.3$ & .005 \\
\hline & & $\begin{array}{l}10 \text { minutes } \\
\text { post }\end{array}$ & $\begin{array}{l}2.5 \\
(1.7)\end{array}$ & $\begin{array}{l}1.2 \\
(2.2)\end{array}$ & $-.17-2.5$ & .082 & $\begin{array}{l}2.1 \\
(1.7)\end{array}$ & $\begin{array}{l}1.5 \\
(1.3)\end{array}$ & $.74-2.3$ & .001 \\
\hline & & $\begin{array}{l}20 \text { minutes } \\
\text { post }\end{array}$ & $\begin{array}{l}2.2 \\
(1.8)\end{array}$ & $\begin{array}{l}1.4 \\
(1.7)\end{array}$ & $.38-2.4$ & .011 & $\begin{array}{l}2.4 \\
(1.8)\end{array}$ & $\begin{array}{l}1.2 \\
(1.4)\end{array}$ & $.37-2.1$ & .009 \\
\hline Tiredness & $6.1(2.3)$ & & $\begin{array}{l}2.2 \\
(2.8)\end{array}$ & $\begin{array}{l}3.8 \\
(3.9)\end{array}$ & $1.5-6.1$ & .004 & $\begin{array}{l}3.2 \\
(3.1)\end{array}$ & $\begin{array}{l}2.8 \\
(3.9)\end{array}$ & $.47-5.2$ & .023 \\
\hline Drowsiness & $5.4(2.8)$ & & $\begin{array}{l}1.5 \\
(2.2)\end{array}$ & $\begin{array}{l}3.9 \\
(3.3)\end{array}$ & $1.9-5.9$ & .001 & $\begin{array}{l}2.8 \\
(2.9)\end{array}$ & $\begin{array}{l}2.6 \\
(3.8)\end{array}$ & $.35-4.9$ & .027 \\
\hline Nausea & $1.5(2.5)$ & & $\begin{array}{l}1.9 \\
(2.9)\end{array}$ & $\begin{array}{l}-.39 \\
(3.3)\end{array}$ & $-2.1-1.4$ & .64 & $\begin{array}{l}1.0 \\
(2.2)\end{array}$ & $\begin{array}{l}0.5 \\
(1.8)\end{array}$ & $-.55-1.6$ & .30 \\
\hline $\begin{array}{l}\text { Lack of } \\
\text { appetite }\end{array}$ & $4.4(3.9)$ & & $\begin{array}{l}4.9 \\
(4.2)\end{array}$ & $\begin{array}{l}-.53 \\
(1.2)\end{array}$ & $-1.3-.23$ & .15 & $\begin{array}{l}4.2 \\
(4.1)\end{array}$ & $\begin{array}{l}0.2 \\
(2.0)\end{array}$ & $-1.1-1.4$ & .79 \\
\hline $\begin{array}{l}\text { Shortness } \\
\text { of breath }\end{array}$ & $2.8(2.4)$ & & $\begin{array}{l}.77 \\
(1.1)\end{array}$ & $\begin{array}{l}2.0 \\
(2.3)\end{array}$ & $.63-3.4$ & .008 & $\begin{array}{l}1.1 \\
(2.0)\end{array}$ & $\begin{array}{l}1.7 \\
(1.7)\end{array}$ & $.66-2.7$ & .004 \\
\hline Depression & $2.2(2.7)$ & & $\begin{array}{l}0 \\
(0.0)\end{array}$ & $\begin{array}{l}2.2 \\
(2.7)\end{array}$ & $.52-3.8$ & .014 & $\begin{array}{l}0.2 \\
(0.4)\end{array}$ & $\begin{array}{l}.19 \\
(2.6)\end{array}$ & $.35-3.5$ & .02 \\
\hline Anxiety & $1.9(2.5)$ & & $\begin{array}{l}0.2 \\
(0.6)\end{array}$ & $\begin{array}{l}1.8 \\
(2.6)\end{array}$ & $.23-3.3$ & .028 & $\begin{array}{l}0.4 \\
(0.7)\end{array}$ & $\begin{array}{l}1.5 \\
(2.1)\end{array}$ & $.27-2.8$ & .02 \\
\hline Wellbeing & $3.4(1.9)$ & & $\begin{array}{l}1.2 \\
(1.8)\end{array}$ & $\begin{array}{l}2.2 \\
(2.5)\end{array}$ & $.66-3.7$ & .009 & $\begin{array}{l}1.8 \\
(1.6)\end{array}$ & $\begin{array}{l}1.6 \\
(2.1)\end{array}$ & $.34-2.9$ & .02 \\
\hline
\end{tabular}

Semi-structured interview

Twelve of the 13 participants completing the study answered open-ended questions about their experiences using both platforms. One participant reported fatigue after the study and was unable to continue. Although participants reported initial difficulties using the hand-held device, they moved around the VR environment easily after a few minutes practice. Two participants required instruction during the VR sessions. All participants described satisfaction, notably pleasure, peacefulness, escape, relaxation and childhood memories, especially using 3D-HMD VR. All participants reported high levels of immersion with most reporting control enabling them to see the full virtual environment. Only two participants were aware of external distractions on the ward. Although three participants reported virtual flora and sky as two-dimensional, all described the virtual environment as being "very realistic". Two participants reported mild non limiting nausea with 3D-HMD VR, while a further two reported initial but transient dizziness in the virtual environment. Finally, most participants stated they would use VR regularly if available for pain relief, emotional respite and relaxation. 
Paired sample T-test analysis showed 3D-HMD VR and 2D computer screen applications produced significant reductions in $\mathrm{CP}$ intensity immediately after both interventions $(1.9 \pm 1.8, \mathrm{P}=.003$ and $1.5 \pm 1.6, \mathrm{P}=.007)$. Participants also reported pain relief at five, 10- and 20-minutes relative to baseline after the 2D computer screen $(P=.005, P=.001$ and $P=.009$ respectively) compared to pain relief with $3 D$ HMD VR relative to baseline at the same time points $(P=.02, P=.08$ and $P=.01$ respectively) (Table 3). At post intervention, participants reported a mean decrease in pain of $43 \%$ using 3D-HMD VR compared to $34 \%$ using the 2D screen application. Linear mixed model analysis showed no significant difference between 3D-HMD VR and 2D screen applications in reductions in CP intensity either immediately after $(-0.38 \pm 1.2,95 \% \mathrm{Cl}:-1.1-0.29, \mathrm{P}$ $=.23$ ), or at five, 10 - and 20-minutes post intervention (Table 3). Although the effects of randomised VR condition sequencing both between and within-subjects on pain scores were not significant immediately after $(P=.07$ and $P=.43$ respectively), at 10- and 20-minutes post interventions, between-subject sequencing effects were significant at five minutes post intervention $(P=.04)$ (Table 4). 
Table 4

A table showing linear mixed model analysis for the effects of VR conditions on a) reported cancer pain intensity immediately, and at five, 10 and twenty minutes, b) reported levels of presence, c) effects of reported levels of presence on post VR condition neuropathic pain intensity and d) as above but showing adjustment for VR condition.

\section{Parameter}

\section{$95 \% \mathrm{Cl}$ (covariate- \\ $\mathrm{t}$ \\ df Significance adjusted)}

\section{Lower bound \\ Upper bound}

Effect of VR conditions on immediate post VR reported pain

intensity (fixed factors - condition, sequence, time)

\begin{tabular}{llllll}
$\cdot$ 3D HMD VR vs 2D screen application & -1.10 & .28 & -1.2 & 13 & .23 \\
\hline$\cdot$ Sequence (between subject) & -3.10 & .14 & -2.0 & 12 & 0.7 \\
- Time (within subject) & -.43 & .95 & .82 & 12 & .43
\end{tabular}

Effect of VR conditions on 5 minutes post VR reported pain intensity

(fixed factors - condition, sequence, time)

-3D HMD VR vs 2D screen application

$-1.2$

.28

$\begin{array}{lll}-1.4 & 12 \quad .20\end{array}$

- Sequence (between subject)

$-3.0-.08$

$-2.2 \quad 20 \quad .04 *$

- Time (within subject)

$-.71 \quad .78$

$\begin{array}{lll}.11 & 12 \quad .92\end{array}$

Effect of VR conditions on 10 minutes post VR reported pain

intensity (fixed factors - condition, sequence, time)

-3D HMD VR vs 2D screen application

$-.59$

1.2

$\begin{array}{lll}.81 & 11 \quad .43\end{array}$

- Sequence (between subject)

$-3.1 \quad .01$

$\begin{array}{lll}-2.2 & 11 \quad .06\end{array}$

- Time (within subject)

$-.42$

1.4

$1.2 \quad 11$

.25

Effect of VR conditions on 20 minutes post VR reported pain

intensity (fixed factors - condition, sequence, time)

$\begin{array}{llllll}\cdot \text { 3D HMD VR vs 2D screen application } & -.64 & .36 & -.62 & 11 & .54 \\ \cdot \text { Sequence (between subject) } & -3.0 & 1.2 & -.91 & 11 & .38 \\ - \text { Time (within subject) } & -.65 & .36 & -.63 & 11 & .54\end{array}$

Effect of VR conditions on post VR reported levels of presence (fixed

factors - condition, sequence, time)

-3D HMD VR vs 2D screen application

- Sequence (between subject)

$\begin{array}{lllll}14.3 & 38.2 & 4.8 & 11 & .001^{\star} \\ -8.7 & 33.2 & 1.4 & 7 & 0.21 \\ -10.2 & 13.7 & .32 & 11 & .75\end{array}$

- Time (within subject)

$-10.2$

13.7

.32

Effect of reported levels of presence on post VR reported pain

intensity (fixed factors - presence, sequence, time)

- IPQ score

- Sequence (between subject)

$\begin{array}{lllll}-.04 & -0.01 & -2.7 & 12 & .02 * \\ -2.8 & .37 & -1.7 & 11 & .12 \\ -.87 & .65 & -0.3 & 14.6 & 0.76\end{array}$

- Time

Effects of VR conditions on reported levels of presence on post VR reported pain intensity (adjusted for VR condition) 


\begin{tabular}{|c|c|c|c|c|c|}
\hline \multirow{3}{*}{$\begin{array}{l}\text { Parameter } \\
\text { • 3D HMD VR vs 2D screen application }\end{array}$} & \multirow{2}{*}{\multicolumn{2}{|c|}{$\begin{array}{l}95 \% \mathrm{Cl} \\
\text { (covariate- } \\
\text { adjusted) } \\
\text { Lower bound } \\
\text { Upper bound }\end{array}$}} & \multirow{3}{*}{$\begin{array}{l}\mathbf{t} \\
\\
1.3\end{array}$} & \multirow{3}{*}{$\begin{array}{l}\text { df } \\
\\
13\end{array}$} & \multirow{3}{*}{$\begin{array}{l}\text { Significance } \\
\\
.22\end{array}$} \\
\hline & & & & & \\
\hline & -.42 & 1.64 & & & \\
\hline - IPQ score & -.07 & -.01 & -2.7 & 14 & $.02 *$ \\
\hline - Sequence (between subject) & -.2 .6 & .52 & -1.4 & 12 & .17 \\
\hline - Time (within subject) & -.30 & .96 & 1.2 & 10 & .28 \\
\hline
\end{tabular}

Effects of 3D HMD VR and 2D screen application on levels of presence

Linear mixed model analysis showed that 3D-HMD VR was associated with significantly greater levels of presence compared to a $2 \mathrm{D}$ screen application (60.7 \pm SD 12.4 versus $34.3 \pm$ SD 17.1, mean 95\% Cl: 16.4-40.7, P = .001) (Supplementary Fig. 2). Furthermore, mixed model analysis also showed that increases in levels of reported presence were associated with greater reductions in pain intensity, regardless of randomised sequencing of VR conditions $(P=.02)$. When VR condition and presence were controlled for, the effect of presence on changes in pain intensity remained significant $(P=.02)(T a b l e 4)$.

Effects of 3D-HMD and 2D screen applications on other symptoms found in palliative care.

Paired T-test analysis showed that 3D-HMD VR and 2D screen applications separately produced significant reductions in some symptoms such as drowsiness $(3.8 \pm 3.3, \mathrm{P}=.001$ versus $2.6 \pm 3.8, \mathrm{P}=.03)$ and shortness of breath $(2.0 \pm 2.3, \mathrm{P}=.008$ versus $1.7 \pm 1.7, P=.004)$. $3 \mathrm{D}$ and $2 \mathrm{D}$ applications also showed significant improvements in wellbeing $(2.2 \pm 2.5, P=.009$ versus 1.6 $\pm 2.1, P=.02$ ) (Table 3 ). Conversely, no significant differences were shown for 3D and 2D applications for other symptoms such as nausea $(P=.64$ versus $P=.30)$, and lack of appetite $(P=.20$ versus $P=.80)$. Like the effects on cancer pain intensity, linear mixed model analysis showed no significant differences in effects between 3D HMD VR and 2D screen applications on all ESAS symptom items.

\section{Discussion}

This study shows the feasibility and acceptability of VR in hospital and home-based settings for people receiving palliative care. Both 3D and 2D platforms were well-tolerated by participants who described positive experiences during and after sessions. Encouragingly, participants reported they would use VR regularly in the future. Given this positive feedback in association with the high completion rates, user comfort, ease of access to affordable VR platforms and encouraging quantitative findings, the estimation of sample size and the prospect of future studies are promising.

Although this feasibility study was not powered to detect differences between groups, our findings indicate that participants with CP report significant decreases in CP intensity during, immediately after and up to 20 minutes after each intervention. However, although linear mixed model analysis showed participants reporting significantly greater levels of presence during 3D HMD VR compared to a 2D screen session, there were no significant differences in reductions of post treatment pain intensity between the two interventions.

Qualitative feedback contributes a deeper evaluation of positive and negative experiences that may explain these findings. First, high levels of user satisfaction engagement and immersion especially using 3D-HMD VR support differences in presence levels between platforms. Second, given the relatively low level of CP intensity at baseline, participants may have had difficulty perceiving changes after each intervention. These latter findings are supported by previous work where more moderate to severe pain at baseline predicts more significant responses to analgesic interventions [30, 31].

Pain-related findings in this study differ to previous work using the same applications and trial protocols in people with spinal cord injury pain (SCI). Here, 3D-HMD VR had significantly greater analgesic effect on SCl pain compared to 2D screen 
applications $(P<.0001)$ where participants showed over $65 \%$ decreases in pain intensity using 3D-HMD VR compared to $43 \%$ in this study [14]. The difference in sample-type may account for this variance. Compared to the long-term stability of SCI and neuropathic pain control, our cancer pain sample were older (71.1 \pm 14.3 versus $54.3 \pm 14.1)$, at varying stages of advanced disease and fluctuating prescriptions and doses of pain medication prior to the study. Our current sample were frailer and reported fluctuating levels of fatigue, pain severity, confusion and pharmacological symptom control. The SCl sample were also younger, more familiar with computer technology and thus were able to familiarise themselves with devices more quickly. In this current study, participants reported similar enjoyment using the 2D applications where they able to sit close to the screen using the bedside table and not feel confined by the headset.

Our findings among ESAS items for palliative care symptoms showed reductions in tiredness, shortness of breath and mood as well as increases in the feeling of wellbeing using both platforms. However, no significant differences were shown for all ESAS item scores when comparing the effects 3D and 2D interventions. Our findings for 3D-HMD VR are comparable to a previous study using similar technology on people reporting CP. Here, Niki and colleagues investigated the effects of 3D HMD VR (Google Earth ${ }^{\circledR}$ ) on symptoms with 20 terminal cancer patients using ESAS as their primary outcome measure. They showed similar findings to our study for all items [32]. Here, both studies report comparably significant reductions in all symptoms except nausea and lack of appetite using 3D-HMD VR. However, given the general symptomatic association between lack of appetite and nausea and the potential for cybersickness these results are explicable.

In the only other study exploring the analgesic effects VR on CP, Mohammad and Ahmed used 3D-HMD VR (Ocean Rift@ or a 'Happy Beach' scene) plus morphine at peak effect compared to morphine alone on women with breast cancer [33]. Their findings show more significant decreases in pain intensity due to the combined analgesic effects of VR and morphine. The greater effects of VR in combination with another form of analgesia are supported by two previous studies where decreases in SCl pain intensity were greater using 3D-HMD VR plus transcranial direct current stimulation compared to VR alone [34, 35].

The duration of VR analgesia is important. Analgesic actions of VR are separated into two types: distraction and neuroplasticity. Distraction represents short-term attentional diversion away from pain towards an alternative stimulus described as 'hijacking' attention, emotion and memory. These effects, depending on the level of presence, may be due to excitability of neuron populations in brain regions associated with pain modulation. Here, healthy participants reporting decreases in pain intensity from painful thermal stimulation during distraction show decreases in thalamic, insular and anterior cingulate cortex activation using functional imaging [36]. Alternatively, neuroplasticity relates to long-term functional and structural changes in neuronal pathways that occur following long-term practice of skills, such as playing a musical instrument or VR use involving interactive real-time simulations of activities. In this study, we investigated only short-term analgesic effects that were assessed during, immediately and up to 20 minutes following each intervention. Attention distraction is the best explanation for the analgesic effect of VR for pain in this population, especially concerning short-term or single session VR use. These immediate effects of VR are shown to be due to temporary activation of top-down pain modulatory pathways via the cingulo-frontal cortex and periaqueductal grey [37].

\section{Limitations}

Due to COVID-19, study recruitment was slower than anticipated. Encouragingly however, another common reason for nonparticipation was the successful management of pain on the ward where potential participants did not have pain at the time of assessment for eligibility. However, although the low sample size may have contributed to reduced significance in analgesic effect between the two groups, the size of the effect following 3D-HMD VR (43\%) is encouraging and suggests a strong albeit temporary analgesic effect. The significant link between immersiveness and analgesia combined with the findings indicating the significantly greater sense of presence with 3D-HMD VR also supports the potential of 3D-HMD VR to produce analgesia in this setting. 
Recruitment was also confounded by the instability of symptoms with advanced disease. Here a participation was planned only for the person to withdraw on the day of the study due to exacerbation of breakthrough symptoms and the effects of pharmacological treatment. Not surprisingly, this was common with people nearing the end of life where only three participated in the trial compared to 10 receiving symptom management. In several instances, family members were not in favour of their relatives participating.

Factors likely to have influenced our findings were the effects of advanced disease and pharmacological treatments on symptoms prior to and during the cross-over trial. Importantly, all participants engaged well with both interventions, however, there were significant differences in levels of concentration and fatigue affecting the completion of self-report measures. Although we only included three short symptom-based questionnaire, participants had difficulty recounting and/or defining symptom severity after both interventions.

\section{Conclusions}

We conclude that using 3D-HMD VR in palliative care pain settings is feasible and acceptable. Virtual experience relieves cancer pain and other symptoms including fatigue. Further research is required to confirm the analgesic effects of VR in larger samples against appropriate control groups.

\section{Declarations}

- Funding: This work was supported by Sydney Vital (grant number 201142)

- Conflicts of interest/Competing interests: The authors declare that they have no conflict of interest

- Availability of data and material: All data, materials and VR hardware and software comply with field standards

- Code availability: Not applicable

- Authors' contributions: All authors contributed to the study conception and design. Material preparation, data collection and analysis were performed by Philip Austin. Writing: original draft (Philip Austin), writing review and editing: (Philip Siddall and Melanie Lovell). Funding acquisition: (Philip Austin and Melanie Lovell). Resources: (Philip Austin). All authors read and approved the final manuscript.

- Ethics approval: The Northern Sydney Local Health District Research Ethics Committee approved this single-site feasibility trial in March 2020, reference 2019/ETH12454

- Consent to participate: All subjects freely gave informed consent to participate in this study

- Consent for publication: All subjects consented to their blinded data to be published in peer-review journals prior to study participation

\section{References}

1. Adam R, Wassell P, Murchie P (2014) Why do patients with cancer access out-of-hours primary care? A retrospective study. Br J Gen Pract 64(619):e99-e104

2. van den Beuken-van Everdingen MHJ, Hochstenbach LMJ, Joosten EAJ, Tjan-Heijnen VCG, Janssen DJA (2016) Update on Prevalence of Pain in Patients With Cancer: Systematic Review and Meta-Analysis. Journal of Pain and Symptom Management;51(6):1070-90.e9

3. Chapman EJ, Edwards Z, Boland JW, Maddocks M, Fettes L, Malia C et al (2020) Practice review: Evidence-based and effective management of pain in patients with advanced cancer. Palliat Med 34(4):444-453

4. Twycross R, Harcourt J, Bergl S (1996) A survey of pain in patients with advanced cancer. J Pain Symptom Manage 12(5):273-282 
5. Caraci F, Crupi R, Drago F, Spina E (2011) Metabolic drug interactions between antidepressants and anticancer drugs: focus on selective serotonin reuptake inhibitors and hypericum extract. Curr Drug Metab 12(6):570-577

6. Chan A, Ng TRD, Yap KY-L (2012) Clinically-relevant anticancer-antidepressant drug interactions. Expert Opinion on Drug Metabolism Toxicology 8(2):173-199

7. Carlson CL (2016) Effectiveness of the World Health Organization cancer pain relief guidelines: an integrative review. Journal of pain research 9:515-534

8. HammondCare. Pain Management in Palliative Care. Sydney: HammondCare; 20142021

9. Pourmand A, Davis S, Marchak A, Whiteside T, Sikka N (2018) Virtual Reality as a Clinical Tool for Pain Management. Curr Pain Headache Rep 22(8):53

10. Cardoş RAl, David OA, David DO (2017) Virtual reality exposure therapy in flight anxiety: A quantitative meta-analysis. Comput Hum Behav 72:371-380

11. Laver KE, Lange B, George S, Deutsch JE, Saposnik G, Crotty M (2017) Virtual reality for stroke rehabilitation. Cochrane Database Syst Rev 11(11):Cd008349

12. Li L, Yu F, Shi D, Shi J, Tian Z, Yang J et al (2017) Application of virtual reality technology in clinical medicine. American Journal of Translational Research 9(9):3867-3880

13. Weech S, Kenny S, Barnett-Cowan M (2019) Presence and Cybersickness in Virtual Reality Are Negatively Related: A Review. Frontiers in Psychology;10(158)

14. Austin PD, Craig A, Middleton JW, Tran Y, Costa DSJ, Wrigley PJ et al (2021) The short-term effects of head-mounted virtual-reality on neuropathic pain intensity in people with spinal cord injury pain: a randomised cross-over pilot study. Spinal Cord 59(7):738-746

15. Kenney MP, Milling LS (2016) The effectiveness of virtual reality distraction for reducing pain: A meta-analysis. Psychology of Consciousness: Theory Research Practice 3(3):199-210

16. Rooney B, Hennessy E (2013) Actually in the Cinema: A Field Study Comparing Real 3D and 2D Movie Patrons' Attention, Emotion, and Film Satisfaction. Media Psychology 16(4):441-460

17. Eldridge SM, Chan CL, Campbell MJ, Bond CM, Hopewell S, Thabane L et al (2016) CONSORT 2010 statement: extension to randomised pilot and feasibility trials. Bmj 355:i5239

18. Courtier N, Gaze S, Armes J, Smith A, Radley L, Armytage J et al (2018) ACTIVE - a randomised feasibility trial study protocol of a behavioural intervention to reduce fatigue in women undergoing radiotherapy for early breast cancer: study protocol. Pilot Feasibility Studies 4(1):85

19. Evans SR (2010) Clinical trial structures. J Exp Stroke Transl Med 3(1):8-18

20. Jin W, Choo A, Gromala D, Shaw C, Squire P (2016) A Virtual Reality Game for Chronic Pain Management: A Randomized, Controlled Clinical Study. Stud Health Technol Inform 220:154-160

21. Fowler CA, Ballistrea LM, Mazzone KE, Martin AM, Kaplan H, Kip KE et al (2019) A virtual reality intervention for fear of movement for Veterans with chronic pain: protocol for a feasibility study. Pilot and Feasibility Studies;5(1):146

22. LaViola JJ (2000) A discussion of cybersickness in virtual environments. SIGCHI Bull 32(1):47-56

23. Farrar JT, Young JP Jr, LaMoreaux L, Werth JL, Poole RM (2001) Clinical importance of changes in chronic pain intensity measured on an 11-point numerical pain rating scale. Pain 94(2):149-158

24. Paice JA (2004) Assessment of Symptom Clusters in People With Cancer. JNCI Monographs 2004(32):98-102

25. Abernethy AP, Shelby-James T, Fazekas BS, Woods D, Currow DC (2005) The Australia-modified Karnofsky Performance Status (AKPS) scale: a revised scale for contemporary palliative care clinical practice [ISRCTN81117481]. BMC palliative care;4:7-

26. Schwind V, Knierim P, Haas N, Henze N. Using Presence Questionnaires in Virtual Reality. Proceedings of the $2019 \mathrm{CHI}$ Conference on Human Factors in Computing Systems; Glasgow, Scotland Uk: Association for Computing Machinery; 2019. p. Paper 360

Page 14/16 
27. Pedroli E, Greci L, Colombo D, Serino S, Cipresso P, Arlati S et al (2018) Characteristics, Usability, and Users Experience of a System Combining Cognitive and Physical Therapy in a Virtual Environment: Positive Bike. Sensors;18(7):2343undefined

28. IBM C (2018) IBM SPSS Statistics for Windows, Version 25.0. IBM Corp, Armonk

29. Kenward MG, Roger JH (2010) The use of baseline covariates in crossover studies. Biostatistics 11(1):1-17

30. Habberstad R, Frøseth TC, Aass N, Bjerkeset E, Abramova T, Garcia-Alonso E et al (2019) Clinical predictors for analgesic response to radiotherapy in patients with painful bone metastases. Ann Oncol 30:v662

31. Förderreuther S, Lampert A, Hitier S, Lange R, Weiser T (2020) The Impact of Baseline Pain Intensity on the Analgesic Efficacy of Ibuprofen/Caffeine in Patients with Acute Postoperative Dental Pain: Post Hoc Subgroup Analysis of a Randomised Controlled Trial. Advances in Therapy 37(6):2976-2987

32. Niki K, Okamoto Y, Maeda I, Mori I, Ishii R, Matsuda Y et al (2019) A Novel Palliative Care Approach Using Virtual Reality for Improving Various Symptoms of Terminal Cancer Patients: A Preliminary Prospective, Multicenter Study. J Palliat Med 22(6):702-707

33. Bani Mohammad E, Ahmad M (2019) Virtual reality as a distraction technique for pain and anxiety among patients with breast cancer: A randomized control trial. Palliat Support Care 17(1):29-34

34. Kumru H, Soler D, Vidal J, Navarro X, Tormos JM, Pascual-Leone A et al (2013) The effects of transcranial direct current stimulation with visual illusion in neuropathic pain due to spinal cord injury: an evoked potentials and quantitative thermal testing study. Eur J Pain 17(1):55-66

35. Soler MD, Kumru H, Pelayo R, Vidal J, Tormos JM, Fregni F et al (2010) Effectiveness of transcranial direct current stimulation and visual illusion on neuropathic pain in spinal cord injury. Brain;133(9):2565-77

36. Bantick SJ, Wise RG, Ploghaus A, Clare S, Smith SM, Tracey I (2002) Imaging how attention modulates pain in humans using functional MRI. Brain;125(2):310-9

37. Valet M, Sprenger T, Boecker H, Willoch F, Rummeny E, Conrad B et al (2004) Distraction modulates connectivity of the cingulo-frontal cortex and the midbrain during pain-an fMRI analysis. Pain;109(3):399-408

\section{Figures}

Figure 1 Cross-over study protocols for 3D HMD and 2D screen VR interventions. A cross-over timeline diagram of the VR protocols that includes VR set-up and baseline questionnaire completion, first VR session, between-VR application washout period, second VR session and post-VR session questionnaire completion.

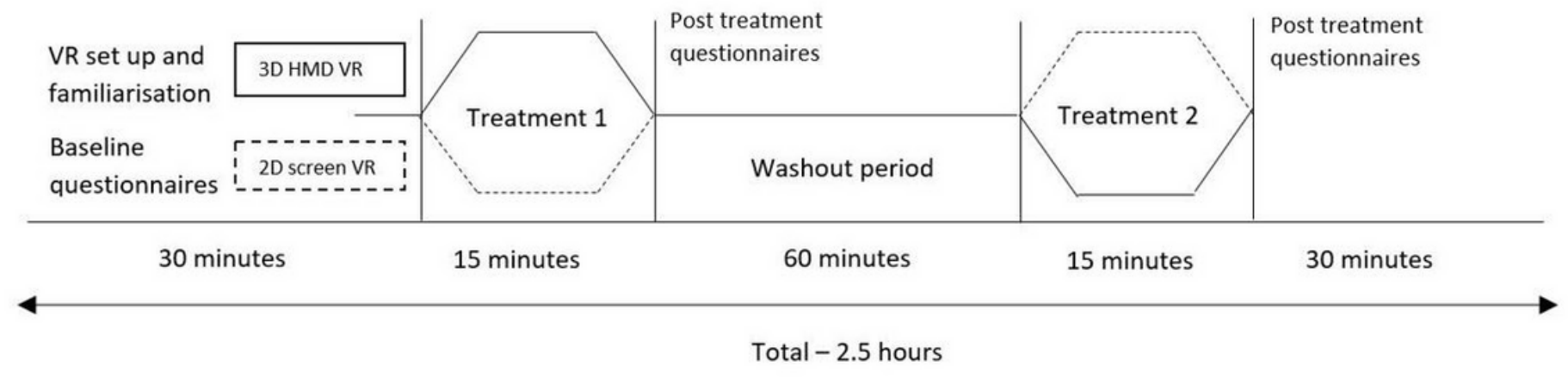

\section{Figure 1}

See image above for figure legend. 
Figure 2 CONSORT flow diagram showing participant screening, enrolment, allocation and assessment of feasibility

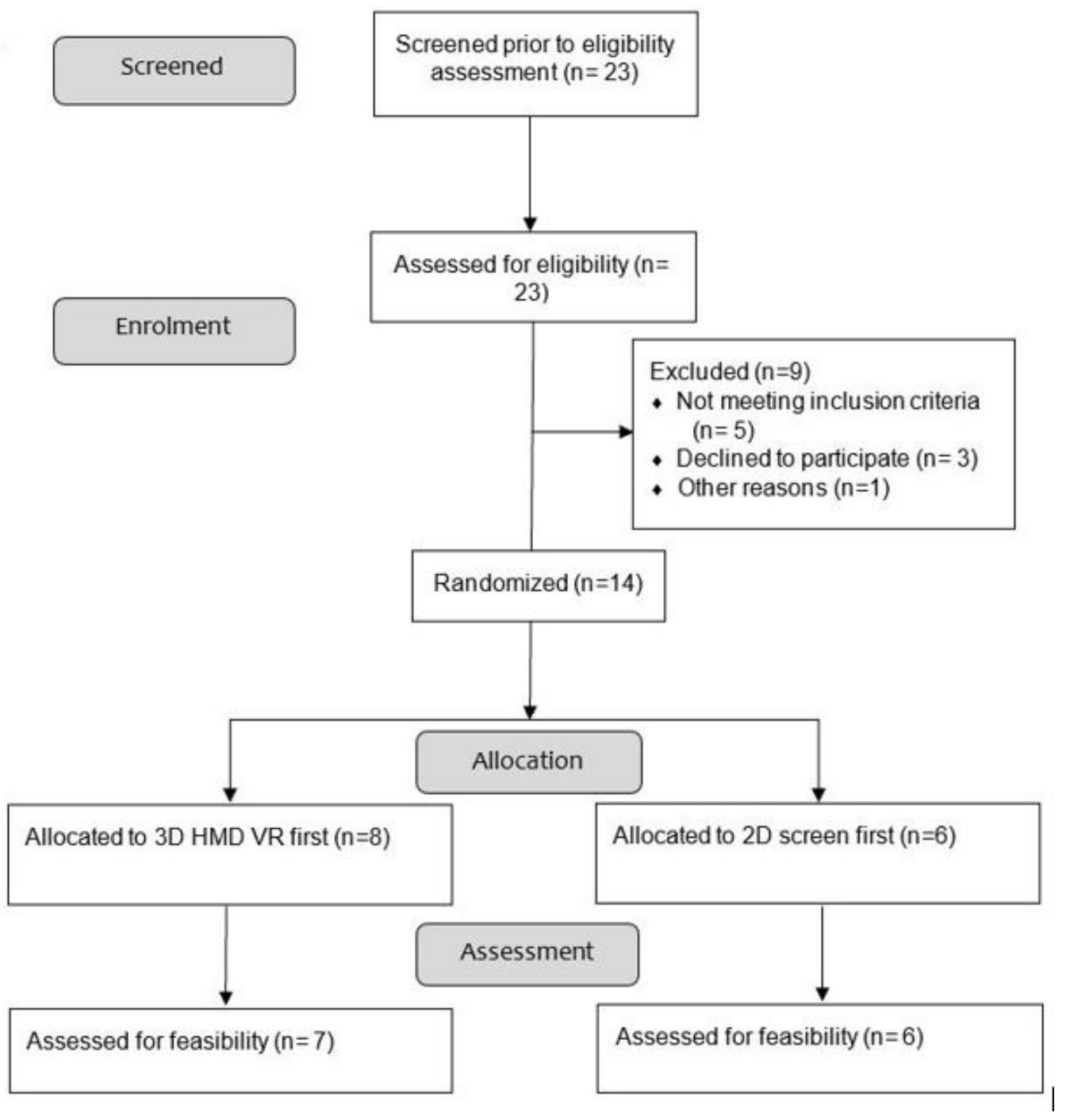

Figure 2

See image above for figure legend.

\section{Supplementary Files}

This is a list of supplementary files associated with this preprint. Click to download.

- CONSORTFeasibilityTrialsChecklist.doc

- SupplFig1SCC300.tiff

- SupplFig2SCC300.tiff 